\title{
Pain associated with health and economic burden in France: results from recent National Health and Wellness Survey data
}

This article was published in the following Dove Press journal:

ClinicoEconomics and Outcomes Research

\author{
Yacine Hadjiat ${ }^{\prime}$ \\ Alain Serrie ${ }^{2}$ \\ Richard Treves ${ }^{3}$ \\ Berangere Chomier ${ }^{\prime}$ \\ Laurent Geranton ${ }^{4}$ \\ Stephane Billon ${ }^{5}$ \\ 'Medical Department, Mundipharma \\ SAS, Paris, ${ }^{2}$ Pain and Palliative Care \\ Department, CHU Lariboisiere, \\ Paris, ${ }^{3}$ Rheumatology Department, \\ CHU Limoges, Limoges, ${ }^{4}$ Public \\ Affairs Department, Mundipharma, \\ ${ }^{5}$ Department of Health Economics, \\ University Paris Dauphine, Paris, \\ France
}

Purpose: This study aimed to estimate the prevalence of pain among French adults and assess the impact of pain on health-related quality of life (HRQoL), activity impairment, and health care resource use (HRU).

Patients and methods: Respondents from the 2015 France National Health and Wellness Survey $(\mathrm{N}=19,173)$ were categorized by self-reported pain (experienced pain in the past 12 months vs no pain) and compared on HRQoL (36-Item Short Form Health Survey version 2: Mental Component Summary, Physical Component Summary, and Short Form-6 Dimensions health utilities), activity impairment (Work Productivity and Activity Impairment questionnaire), employment status, and HRU (health care provider visits, emergency room visits, and hospitalizations). Bivariate analyses examined differences between pain groups stratified by age, sex, income, and Charlson comorbidity index (CCI) scores.

Results: Pain prevalence was 20.2\% ( $\mathrm{n}=4007)$. Mean Physical Component Summary decrements with pain ranged from 3.4 to 8.1 points among those aged $<35$ years to those aged $45-54$ years, respectively. Results for Mental Component Summary and Short Form-6 Dimensions scores followed similar patterns. Regardless of income, sex, or CCI group, pain was associated with significant decrements on all HRQoL measures (for all, $p<0.05$ ). The impact of pain on activity impairment was lowest among those $<35$ years; this impact was higher in middle age and then tapered off among those aged $\geq 75$ years. Pain was associated with greater activity impairment and more health care provider visits across income, sex, and CCI groups (for all, $p<0.05$ ). Generally, emergency room visits were more common among those with pain across age, sex, and CCI, but they were only significantly associated with pain in the lower income group $(p<0.01)$. Pain was associated with significantly more hospitalizations across age and income groups.

Conclusion: Results suggest pain negatively affects HRQoL, activity impairment, and HRU across demographic subgroups. These findings help underscore the considerable health and economic burden of pain in France.

Keywords: activity impairment, health care resource use, health-related quality of life, prevalence

\section{Introduction}

Pain is an important determinant of health-related quality of life (HRQoL). For example, pain and psychiatric conditions, such as post-traumatic stress disorder and depression, often co-occur, ${ }^{1,2}$ highlighting the link between pain and poorer mental health. Chronic pain has also been associated with difficulty with sleeping and interpersonal interactions, as well as mobility impairments. ${ }^{3}$ Moreover, in a multinational cross-sectional population survey, adults who self-reported experiencing pain in the past month due to
Correspondence: Yacine Hadjiat Medical Department, Mundipharma, 12 Marina View, 22-0I Asia Square Tower 2, Singapore 018961

Tel +6565 II II 65

Fax +6565111167

Email yacine.hadjiat@mundipharma. com.sg 
one of several medical conditions (e.g., surgery, arthritis, etc.) had lower HRQoL than controls, which exceeded minimally important differences (MIDs), as measured by scores on the Medical Outcomes Study Short Form 12-Item Health Survey (SF-12) Mental Component Summary (MCS) and Physical Component Summary (PCS) and Short Form-6 Dimensions (SF-6D) health utilities. ${ }^{4}$

In addition to its impact on HRQoL, pain can have substantial economic consequences for patients and society. Adults who reported experiencing a pain condition in the prior month were found to have greater work productivity loss and health care resource use (HRU), including health care provider (HCP) visits, emergency room (ER) visits, and hospitalizations, than controls. ${ }^{4} \mathrm{~A}$ previous systematic review suggested that the socioeconomic burden of chronic pain in the European Union (EU) is greater than that attributed to other serious health conditions, such as heart disease or diabetes..$^{5}$ Indirect costs due to work impairment, early retirement, and disability appear to account for much of the financial burden in this region, with hospitalizations being the greatest factor affecting direct costs.

With respect to France in particular, data on the humanistic and economic burden of specific types of pain have been reported. For instance, according to a cross-sectional observational study of adults diagnosed with fibromyalgia, patients' Brief Pain Inventory scores indicated that pain interfered with daily activities, work, sleep, and enjoyment of life. ${ }^{6}$ In that study, work productivity loss contributed almost $90 \%$ of the total costs incurred by patients with fibromyalgia; HCP visits and prescription medications accounted for most of the direct costs. In a cross-sectional survey of the general adult population in France, those with neuropathic pain reported lower HRQoL on the MCS and PCS and higher HRU than controls and adults with non-neuropathic pain. ${ }^{7}$ Adults with chronic lower back pain reported poor HRQoL (MCS and PCS scores), with physical therapy and hospitalizations accounting for the largest proportion of direct costs, in a retrospective observational cohort study of French patients who visited general practitioners. ${ }^{8}$

As noted previously, most studies in this area have focused on specific pain conditions, whereas research is lacking on the broader impact of pain in France. The few prior largescale surveys on this topic were based on data collected in 2008 or earlier. ${ }^{9,10}$ In one of these studies, data from French participants were aggregated together with respondents from four other EU nations and then categorized by pain severity level, which precluded a determination of the prevalence and burden of pain unique to the general adult population in France. Whether the impact of pain on outcomes varies by demographic subgroups is not well understood. Further clarification regarding the aforementioned issues is necessary to facilitate the ability of HCPs and policymakers in France to develop more effective strategies to address the health and economic burden attributed to pain.

\section{Objectives}

The aims of this study were two-fold. First, the prevalence of pain among the general adult population in France, stratified by age group, was estimated using recent data. Second, the impact of pain on adults in France in terms of HRQoL, employment, impairment to daily activities, and HRU was investigated.

\section{Materials and methods \\ Data source}

For the current study, data were analyzed from the 2015 France National Health and Wellness Survey (NHWS; Kantar Health, NY, USA; $N=19,173$ ), which is a self-report, internetbased survey of adults aged $\geq 18$ years. The NHWS employs a stratified random sampling framework to ensure a representative sample. Specifically, participants are selected to match the age and sex distributions of the general adult population in France using estimates from the International Database of the US Census Bureau. Potential NHWS respondents are identified using Lightspeed Research and affiliated opt-in online survey research panels. A total of 176,242 invitations were sent for the France 2015 NHWS, yielding a response rate of roughly $11 \%$. All participants provided their informed consent online, prior to completing the NHWS. The NHWS was approved by the Pearl Institutional Review Board (Lebanon, NJ, USA). NHWS data will be made available upon request, for purposes of study verification or replication.

\section{Measures}

\section{Independent variables}

\section{Pain experience}

NHWS respondents were asked whether they experienced a pain condition within the prior 12 months. This variable was then dichotomized (i.e., pain vs no pain).

\section{Demographic characteristics}

Age was categorized as $<35$ years and then in 10-year increments as follows: $35-44,45-54,55-64,65-74$, and $\geq 75$ years. Respondents were also asked to indicate whether they were male or female. Household income was categorized by an approximate median split ( $€<30,000$ vs $€ \geq 30,000$ ). 
Those who declined to answer were excluded from the analyses that included income.

\section{Comorbidity burden}

The Charlson comorbidity index (CCI) weights and then sums the self-reported presence of several chronic health conditions, including the following assessed in the NHWS: diabetes, chronic pulmonary disease, dementia, myocardial infarction, human immunodeficiency virus/acquired immunodeficiency syndrome, metastatic tumor, lymphoma, leukemia, any tumor, moderate-to-severe renal disease, hemiplegia, mild liver disease, ulcer disease, connective tissue disease, cerebrovascular disease, peripheral vascular disease, and congestive heart failure. ${ }^{11}$ Higher CCI scores signify a greater comorbidity burden for a given individual. For this study, CCI scores were categorized as CCI $=0,1-2$, $3-4$, or $\geq 5$ as Charlson et al found these cut-offs predicted differences in mortality rates (mortality rates of $12 \%, 26 \%$, $52 \%$, and $85 \%$ were associated with CCI scores of $0,1-2$, $3-4$, and $\geq 5$, respectively). ${ }^{11}$ CCI scores are most often used to adjust for the confounding influence of non-psychiatric comorbid conditions on health outcomes.

\section{Outcome variables}

\section{Health-related quality of life}

The Medical Outcomes Study Short Form 36-Item Health Survey version 2, a widely-used questionnaire, provided three separate HRQoL metrics: 1) MCS scores, which measure mental and emotional health, 2) PCS scores, which measure physical health, and 3) SF-6D health utilities, a preference-based index score. ${ }^{12,13}$ The MCS and PCS have a theoretical range of $0-100$, whereas scores on the SF-6D can range from 0 to 1 . Higher scores on these measures indicate better HRQoL. MIDs are indicated by differences of 3.0 points for MCS and PCS scores and 0.04 points for SF-6D scores. ${ }^{14,15}$

\section{Activity impairment}

Activity impairment was measured with the Work Productivity and Activity Impairment questionnaire, general health version. ${ }^{16}$ Respondents reported the percentage of daily activity impairment due to their health in the past week. Scores can range from $0 \%$ to $100 \%$, with higher values indicating greater impairment in non-work daily activities. To measure the impact of pain on taking part in the labor force, respondents were categorized as being employed (part-time, full-time, or self-employed) or not employed, based on their self-reported employment status.

\section{Health care resource use}

Respondents were asked to report their HRU within the prior 6 months due to any cause. Specifically, NHWS data on the number of HCP visits, ER visits, and hospitalizations were collected.

\section{Statistical analyses}

The proportion of respondents who reported experiencing pain in the prior 12 months was compared across age, sex, income, and CCI categories, using chi-square tests for the percentage of those who indicated at least 1 ER visit and the percentage with at least 1 hospitalization. Chi-square tests were also used to evaluate differences between pain groups in employment status and labor force participation by age category.

Independent-samples $t$-tests were conducted to examine differences between those with and without pain on MCS, PCS, SF-6D, activity impairment, and the number of HCP visits. These analyses were performed separately for age, sex, income, and CCI categories, while collapsing across the non-focal categories (e.g., analyses by age group collapsed across sex, income, and CCI categories; analyses by sex collapsed across age, income, and CCI categories, and so forth). Those who reported pain but declined to provide income data $(n=299)$ were excluded from the analyses for income group.

\section{Results \\ Sample characteristics and pain prevalence}

Out of the total NHWS sample, just under half of respondents $(48.3 \%)$ were male, and the average age was 47.9 years. Pain was reported by $20.2 \%$ of the sample $(n=4007)$, and it was more commonly reported by women than men $(23.1 \%$ vs $17.1 \%, p<0.01$, Figure 1). The prevalence of self-reported pain varied according to age $(p<0.001)$. Specifically, pain was reported by $18.1 \%$ of those $<35$ years, which increased to $22.8 \%$ and $23.7 \%$ among those aged $45-54$ years and 55-64 years, respectively; however, pain was reported by only $15.2 \%$ of those aged $\geq 75$ years (Figure 1 ). Pain was more common among those with lower household income (<€30,000;22.6\% vs $\geq € 30,000 ; 19.0 \%, p<0.01$, Figure 1).

Most respondents $(84 \%, n=16,059)$ had CCI scores of 0 (Table 1). The proportion of respondents who reported pain was higher with increasing CCI score category. Very few respondents had CCI scores of 3-4 or $\geq 5$.

\section{Health-related quality of life}

PCS, MCS, and SF-6D scores were significantly lower among those reporting pain across all age groups, relative to those 

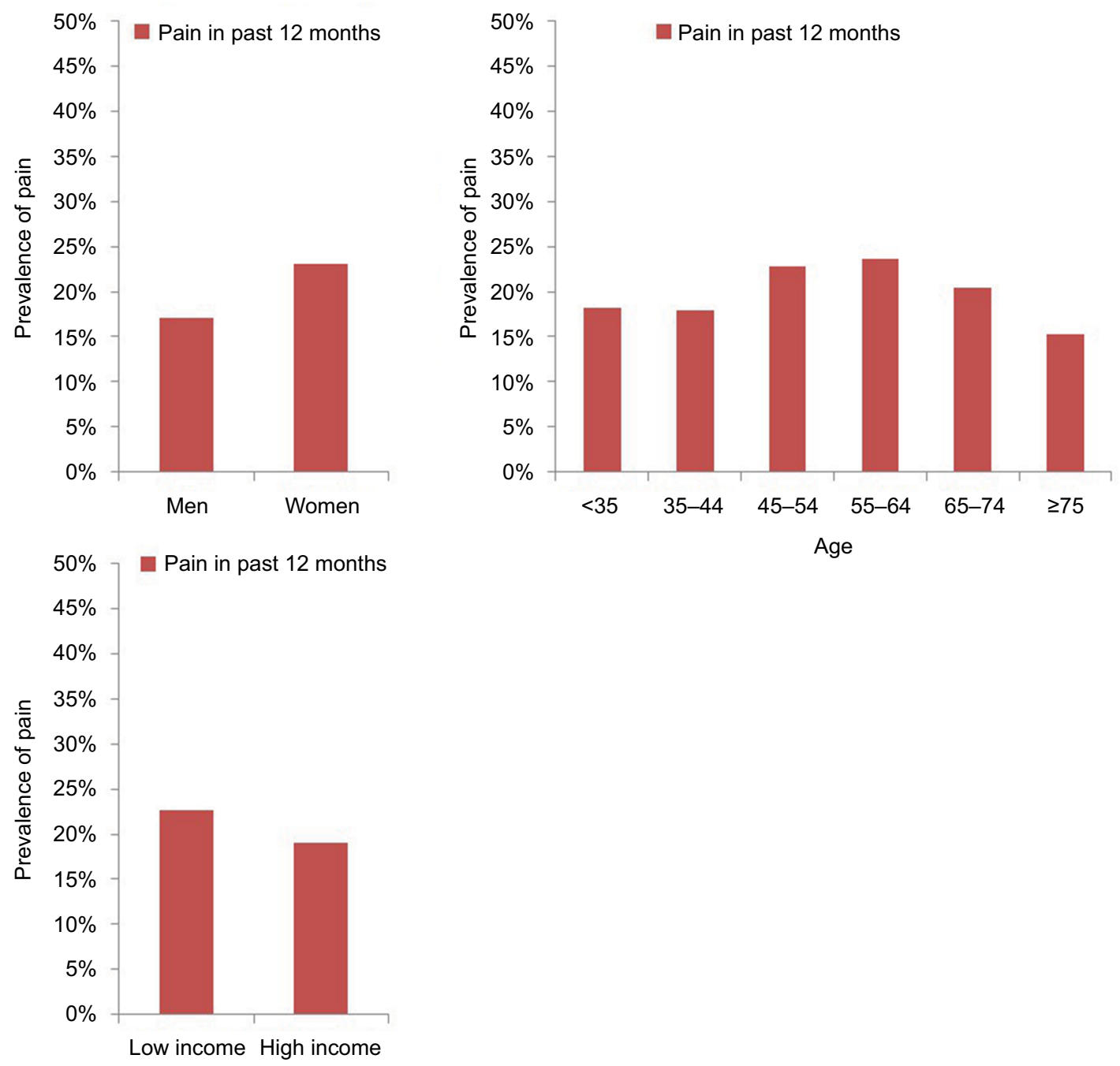

Figure I Prevalence of pain in the past 12 months, according to sex, age, and income level.

Table I Respondents according to sex, presence of pain, and comorbidity category

\begin{tabular}{llllll}
\hline Subsample & \multicolumn{2}{l}{ Women } & & & Men \\
\cline { 2 - 3 } \cline { 5 - 6 } & No pain & Pain & & No pain & Pain \\
\hline Overall & 7657 & 2408 & & 7509 & 1599 \\
CCI: 0 & 6865 & 1897 & & 6221 & 1076 \\
CCI: $1-2$ & 745 & 464 & & 1149 & 433 \\
CCI: $3-4$ & $4 I$ & 33 & & 92 & 62 \\
CCI: $\geq 5$ & 6 & 14 & & 47 & 28 \\
\hline
\end{tabular}

Abbreviation: $\mathrm{CCl}$, Charlson comorbidity index.

reporting no pain, with the exception of MCS scores among those aged $\geq 75$ years ( 2.9 points lower, $p=0.057$ ) (Figures 2 and 3). The magnitude of the negative impact on HRQoL associated with pain varied with age. Compared with the no pain group, mean PCS decrements of 3.4 points among those aged $<35$ years, 8.1 points among those aged $45-54$ years, and 5.4 points among the elderly (aged $\geq 75$ years) were found. All of these differences exceeded the MID of 3.0 points for this measure. The magnitude of the associations between pain and MCS and SF-6D scores followed a similar, although less pronounced pattern; specifically, the smallest impact was found among those $<35$ years, with higher and fairly stable impairment in middle age, followed by a modest reduction in the impact of pain among those aged $\geq 75$ years. The relationship between pain and HRQoL was stable, regardless of income, as pain was associated with significant decrements in PCS, MCS, and SF-6D scores across income groups (for all, $p<0.01$; Figures 2 and 3 ).

HRQoL was worse among those with pain, with consistent results across sex and CCI categories. Specifically, MCS scores were $\sim 3.5$ points lower, overall, compared with the no pain group (Figure 4). PCS scores were more than 6.0 points 

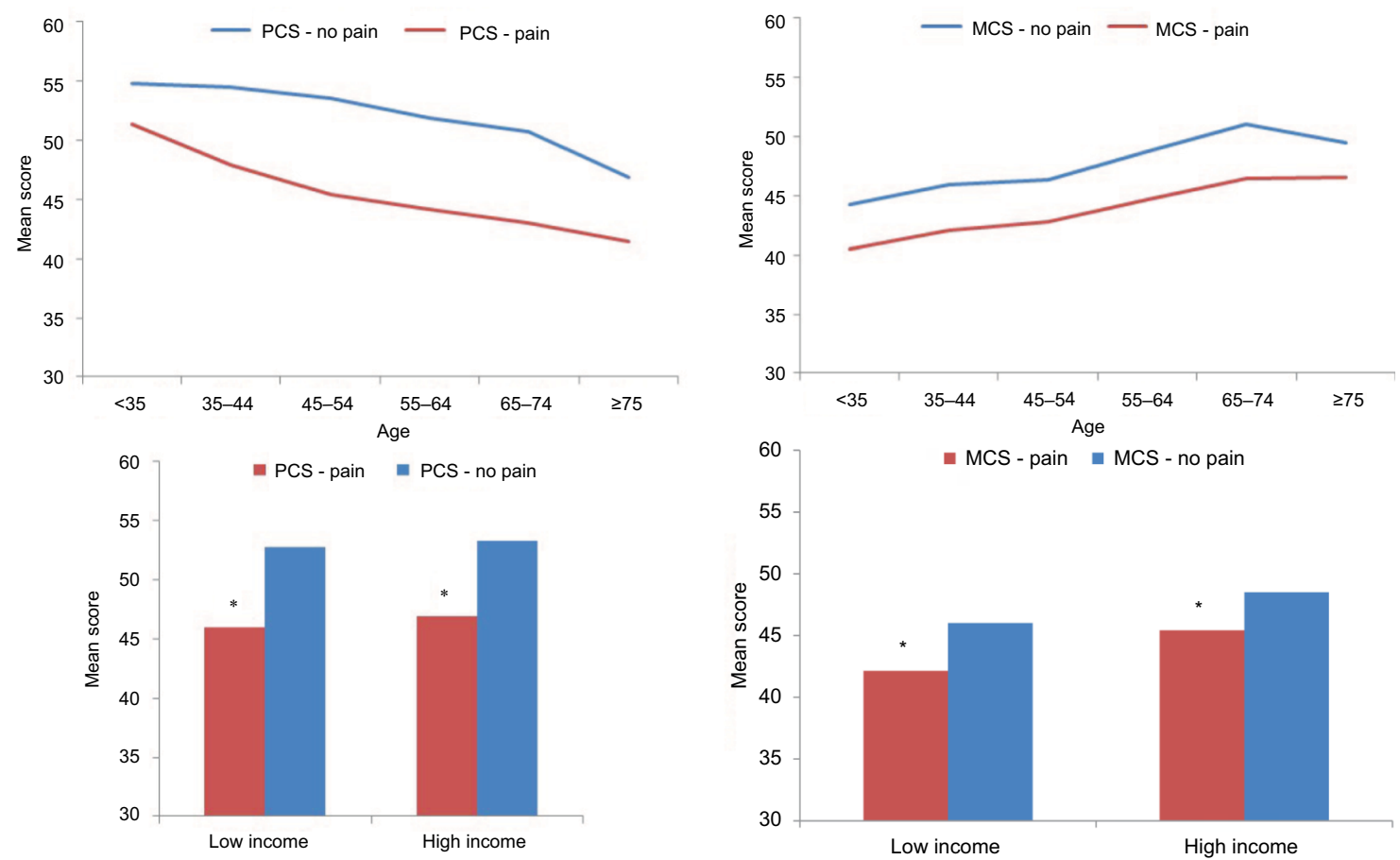

Figure 2 Mean mental and physical component summary scores, according to pain, age group, and income level.

Notes: Pairwise tests between pain and no pain were significant for PCS for ages $<35,35-44,45-54,65-74$, and $\geq 75$; for MCS, for ages $<35,35-44,45-54,65-74$. *p $<0.05$. Abbreviations: MCS, mental component summary; PCS, physical component summary.

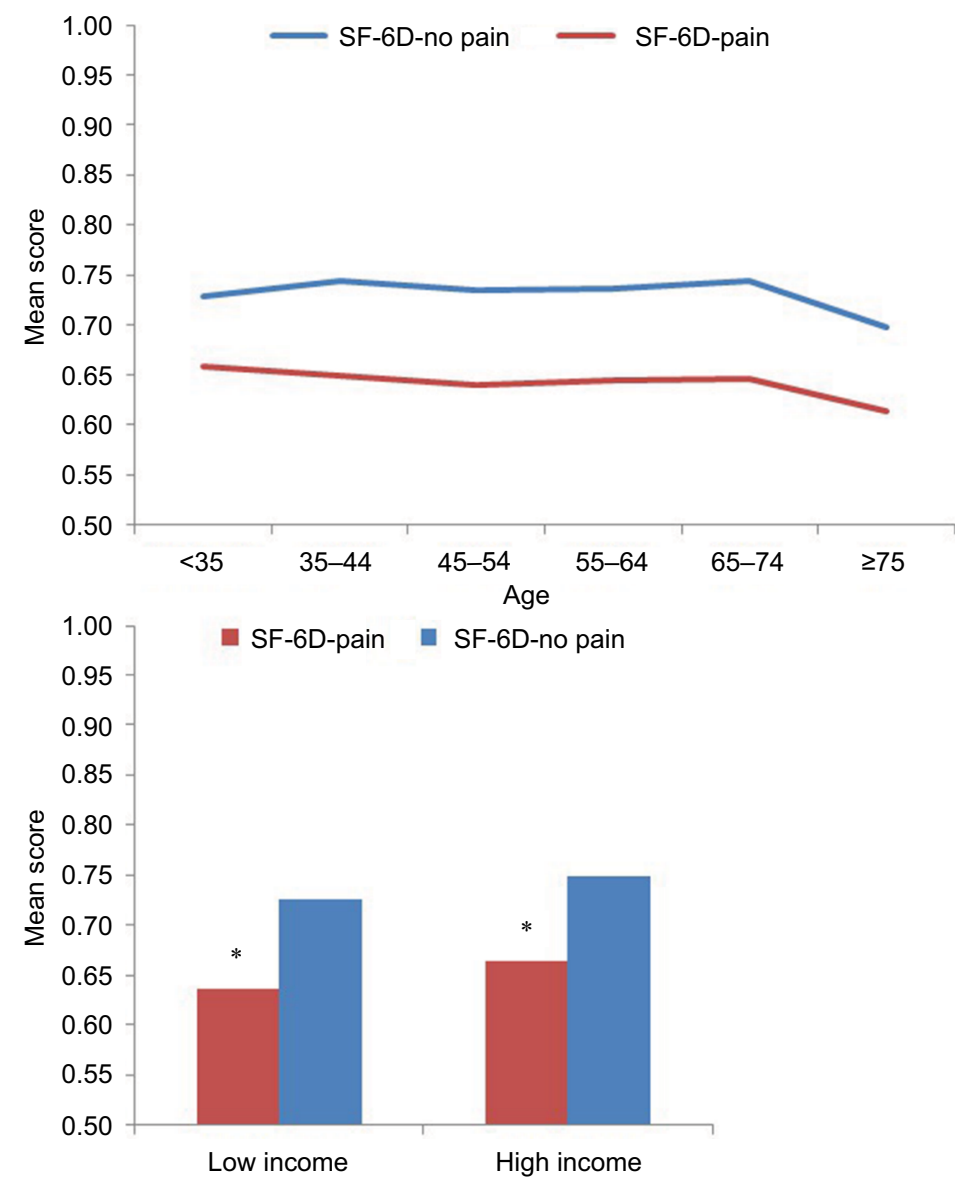

Figure 3 Mean SF-6D health utility score, according to pain, age group, and income level.

Notes: Pairwise tests between pain and no pain were significant for ages $<35,35-44,45-54,65-74$, and $\geq 75$. ${ }^{*} p<0.05$.

Abbreviations: $\mathrm{CCl}$, Charlson comorbidity index; SF-6D, Short Form 6-Dimensions. 

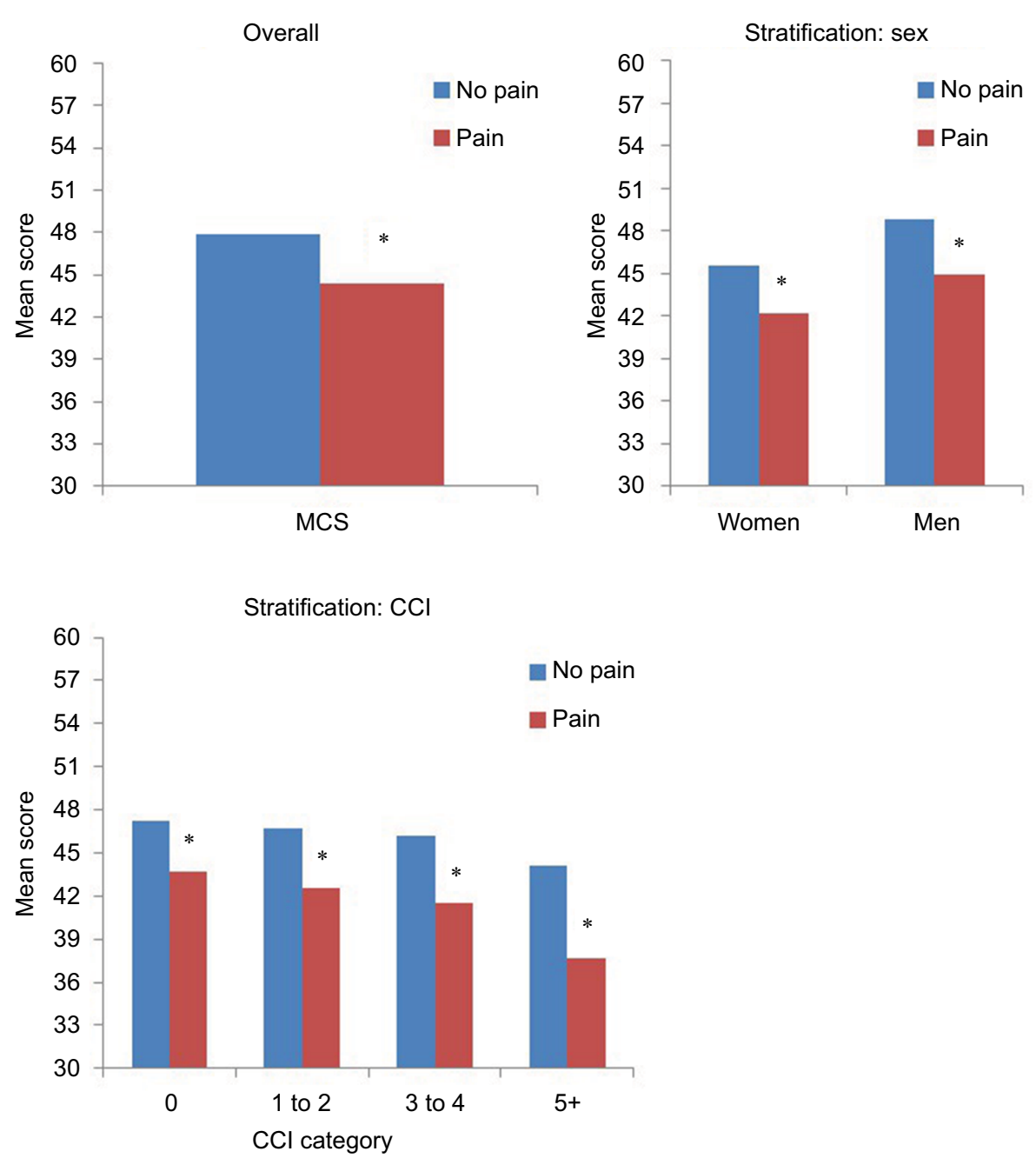

Figure 4 Mental component summary scores, according to pain, sex, and $\mathrm{CCl}$ category. Notes: Higher $\mathrm{CCl}$ categories had small sample sizes. ${ }^{*} p<0.05$.

Abbreviations: $\mathrm{CCl}$, Charlson comorbidity index; $\mathrm{MCS}$, mental component summary.

lower (Figure 5), and SF-6D health utilities were 0.09 points lower, overall, than the no pain group (Figure 6). These differences exceeded the MIDs of 3.0 points (MCS and PCS) and 0.04 points (SF-6D) for these three measures.

\section{Activity impairment}

Similar to results for HRQoL, the impact of pain on activity impairment was lowest among those $<35$ years; this impact was higher and fairly stable in middle age and slightly lower among those aged $\geq 75$ years (Figure 7). The relationship between pain and activity impairment was also stable across both levels of income. Activity impairment was approximately twice as high among those with pain, compared with those without pain, across sex and CCI score categories (Figure 8). Pain was associated with a lower probability of being employed or in the labor force among those aged $\leq 55$ years (for each, $p<0.05$, Figure 9).

\section{Health care resource use}

There was an increasing trend in terms of number of HCP visits across age groups, but differences between the pain and no-pain groups were relatively small (Figure 10). Pain was associated with more HCP visits in each of the income strata (for each, $p<0.01$ ). ER visits were more common among those with pain in most age groups, but generally trended lower with increasing age (Figure 11). ER visits were significantly associated with pain in the lower income group $(p<0.01)$ but not significantly associated with pain among those with higher income $(p=0.09)$. Hospitalizations were also significantly associated with pain across most age groups, as well as across levels of income (Figure 11).

Respondents with pain also had significantly more HCP visits, on average, by sex and most CCI categories (Figure 12; for all except CCI scores of $3-4, p<0.05$ ). Pain was also significantly associated with visiting the ER for 

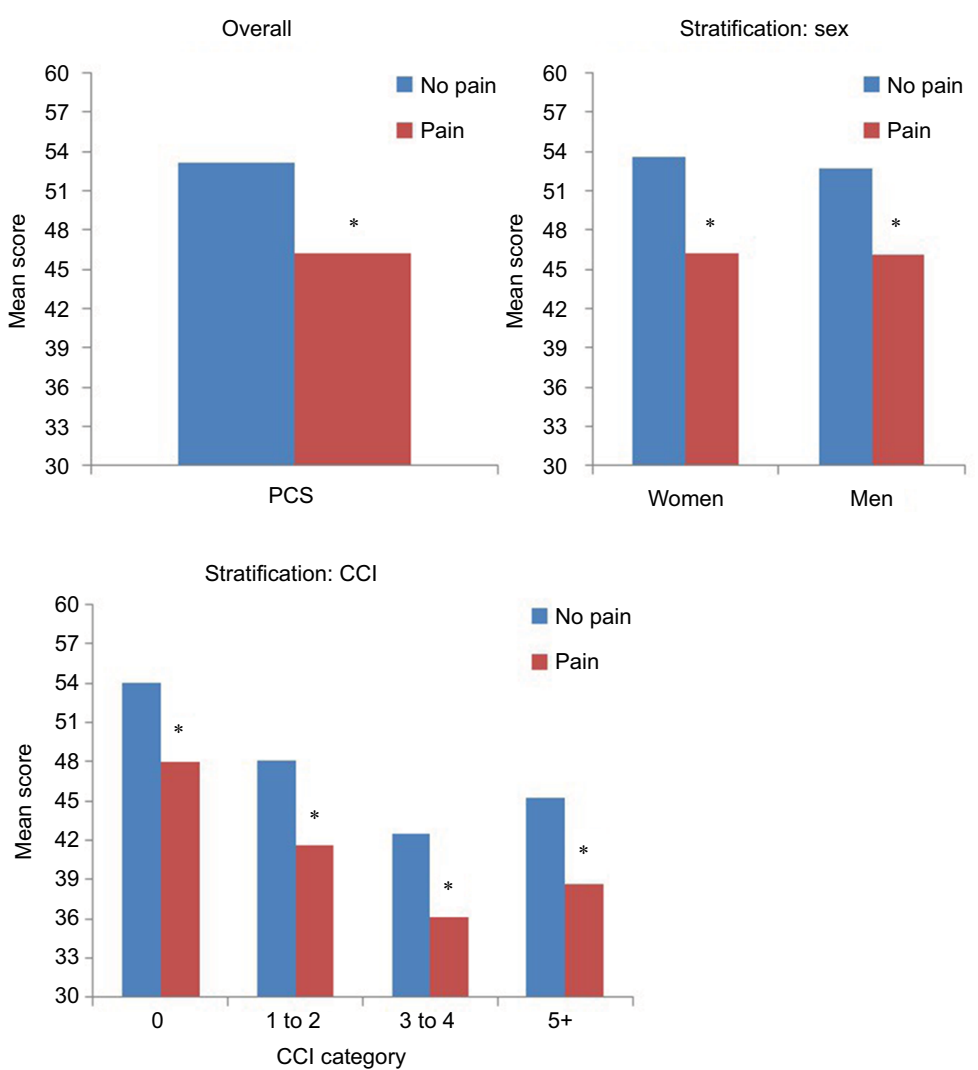

Figure 5 Physical component summary scores, according to pain, sex, and CCl category. Notes: Higher $\mathrm{CCl}$ categories had small sample sizes. ${ }^{*} p<0.05$.

Abbreviations: $\mathrm{CCl}$, Charlson comorbidity index; PCS, physical component summary.
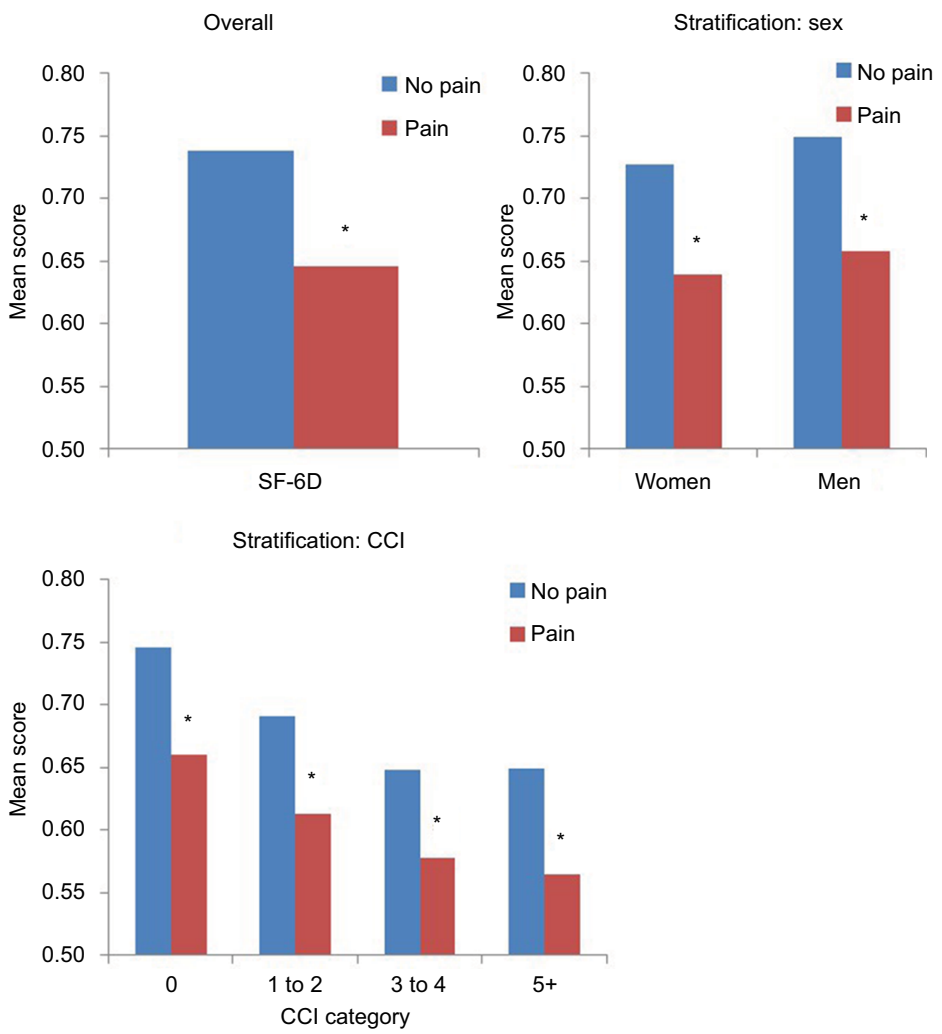

Figure 6 SF-6D health utility scores, according to pain, sex, and CCl category.

Notes: Higher $\mathrm{CCl}$ categories had small sample sizes. ${ }^{*} p<0.05$.

Abbreviations: $\mathrm{CCl}$, Charlson comorbidity index; SF-6D, Short Form 6-Dimensions. 

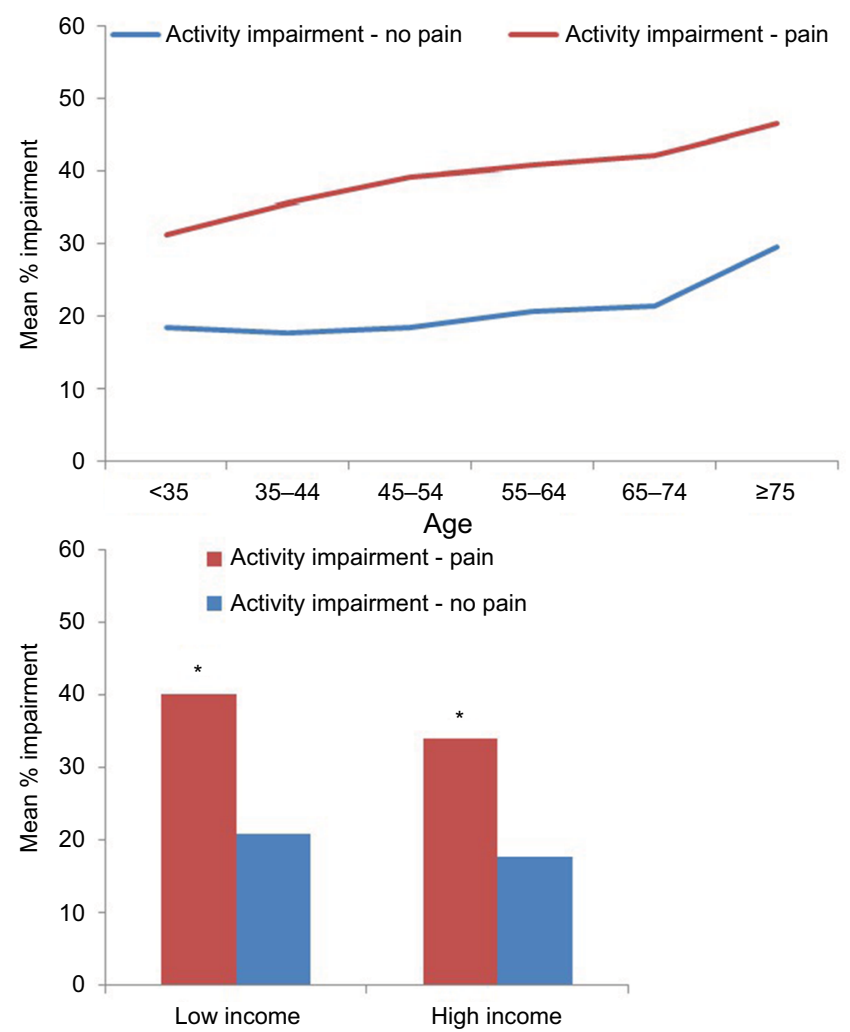

Figure 7 Mean activity impairment (WPAl), according to pain, age group, and income level.

Notes: Pairwise tests between pain and no pain were significant for ages $<35,35-44,45-54,65-74$, and $\geq 75$. ${ }^{*} p<0.05$.

Abbreviation: WPAI, Work Productivity and Activity Impairment.
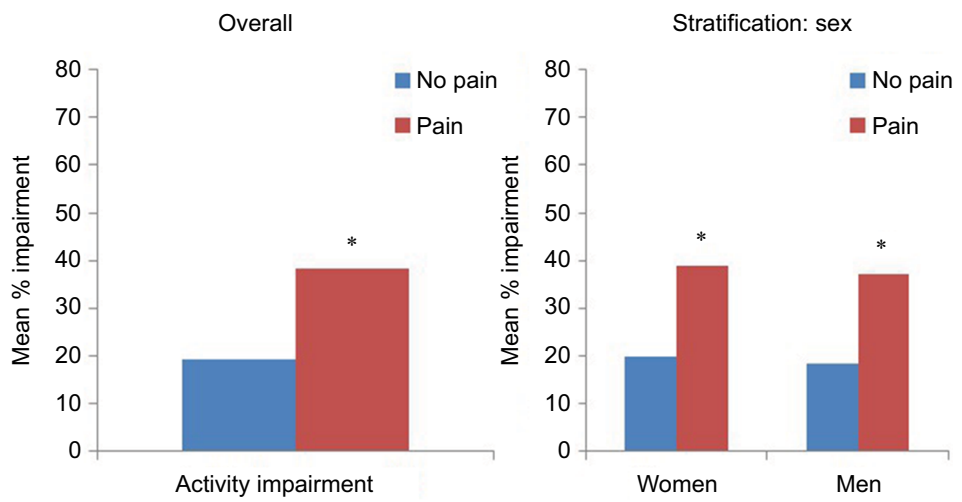

Stratification: CC

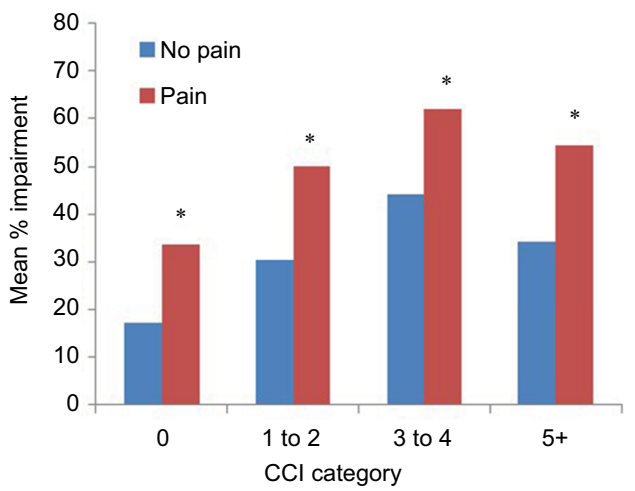

Figure 8 Mean activity impairment (WPAI), according to pain, sex, and $\mathrm{CCl}$ category.

Notes: Higher $\mathrm{CCl}$ categories had small sample sizes. ${ }^{*} p<0.05$.

Abbreviations: $\mathrm{CCl}$, Charlson comorbidity index; WPAl, Work Productivity and Activity Impairment. 


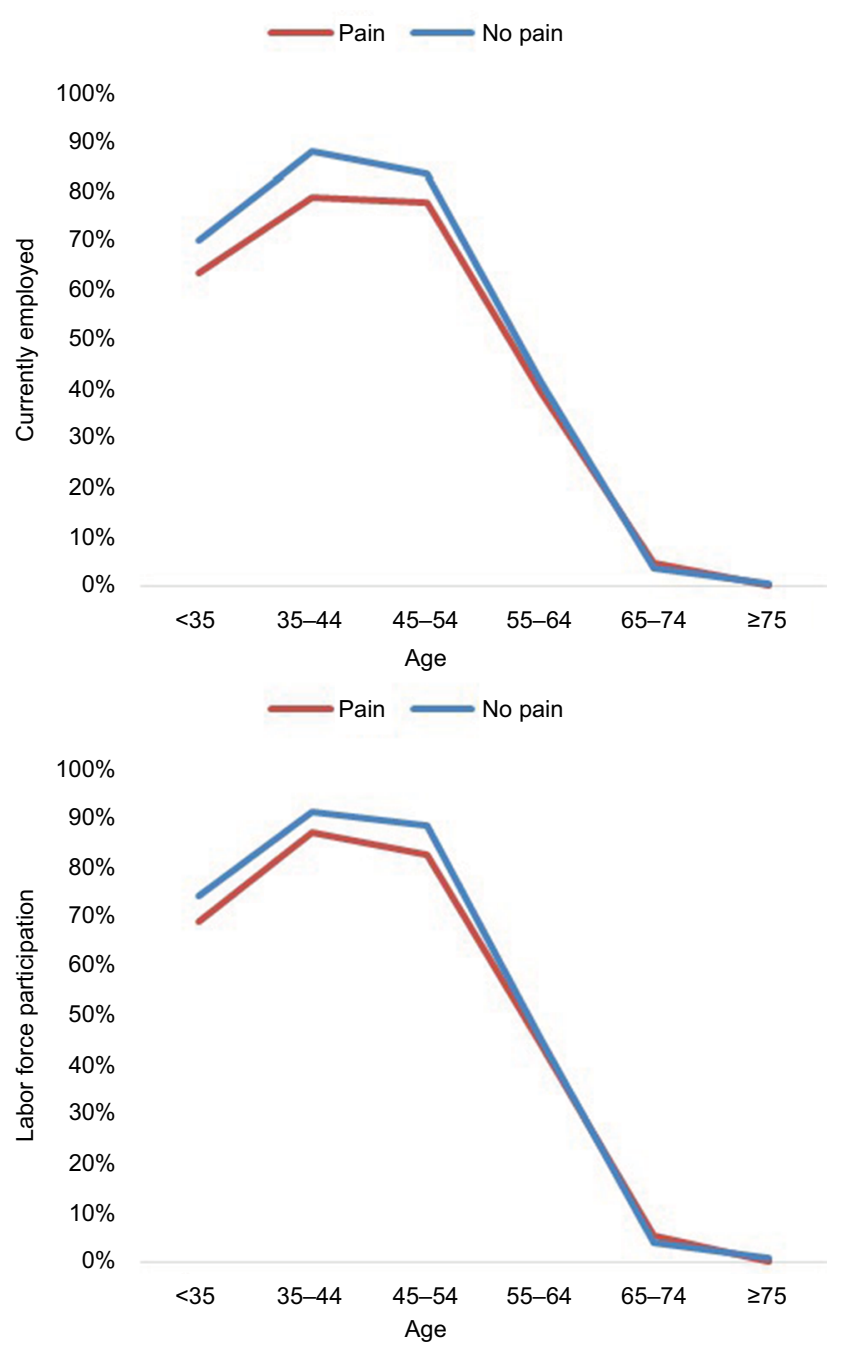

Figure 9 Prevalence of employment, according to pain and age group, in past 12 months.

Notes: Pairwise tests between pain and no pain were significant for ages $<35$, 35 44 , and $45-54$ at $p<0.05$.

both males and females and among those with CCI scores of 0 or 1-2 (for each, $p<0.05$; Figure 13). Those with pain were also more likely to be admitted to the hospital across sex and most CCI categories (for all except CCI scores of $3-4, p<0.05$; Figure 14).

\section{Discussion}

The purpose of this study was to estimate the prevalence of pain according to age group and assess the burden of pain in terms of HRQoL, employment, daily activity impairment, and HRU, among the general adult population in France. The current study was able to fill important gaps in the available literature. Specifically, the few studies that have evaluated the prevalence and impact of pain in France were not based on recent data. ${ }^{9,10}$ In contrast, this study used data collected in 2015 from a large-scale population survey. Furthermore, by
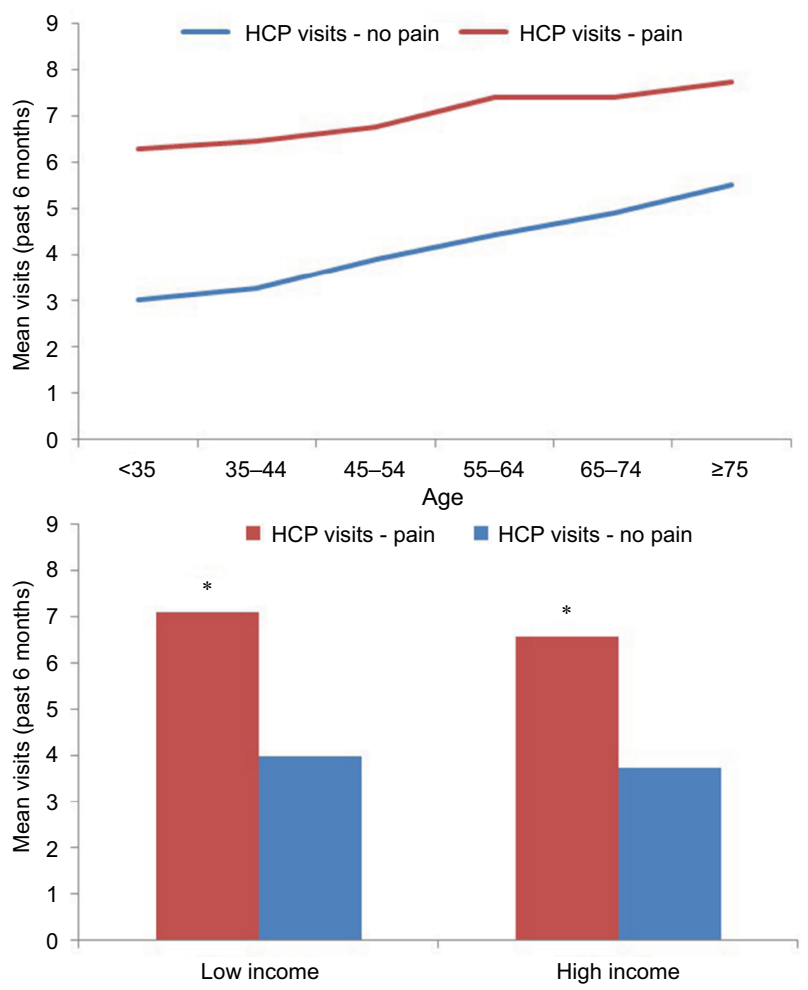

Figure 10 Mean number of health care provider visits in past 6 months, according to pain, age group, and income level.

Notes: Pairwise tests between pain and no pain were significant for ages $<35$, 3544, 45-54, 55-64, 65-74, and $\geq 75$. * $p<0.05$.

Abbreviation: $\mathrm{HCP}$, health care provider.

considering a broad array of relevant outcomes, the present study was able to more comprehensively clarify the nature of the burden ascribed to pain in this region.

Overall, the prevalence of pain in France was high. Particularly, results revealed that approximately 1 in 5 adults in France reported experiencing pain in the prior 12 months. This estimate was in line with those provided by prior studies with EU samples, ${ }^{4,17}$ as well as within the ranges calculated from samples of French adults.., 18

Results suggested that adults in France who have pain suffer from clinically meaningful reductions in both mental and physical HRQoL. These findings were aligned with previous studies demonstrating the connection between experiencing pain and having poor mental health, ${ }^{1,2}$ as well as lower HRQoL. ${ }^{4}$ Moreover, findings indicated that pain was associated with lower employment and labor force participation, increased activity impairment, and more HRU. These findings were consistent with prior research in which adults with chronic pain reported impairments to daily activities, sleeping, and mobility, ${ }^{3}$ as well as reduced work productivity and more ER visits, HCP visits, and hospitalizations. ${ }^{4}$ 

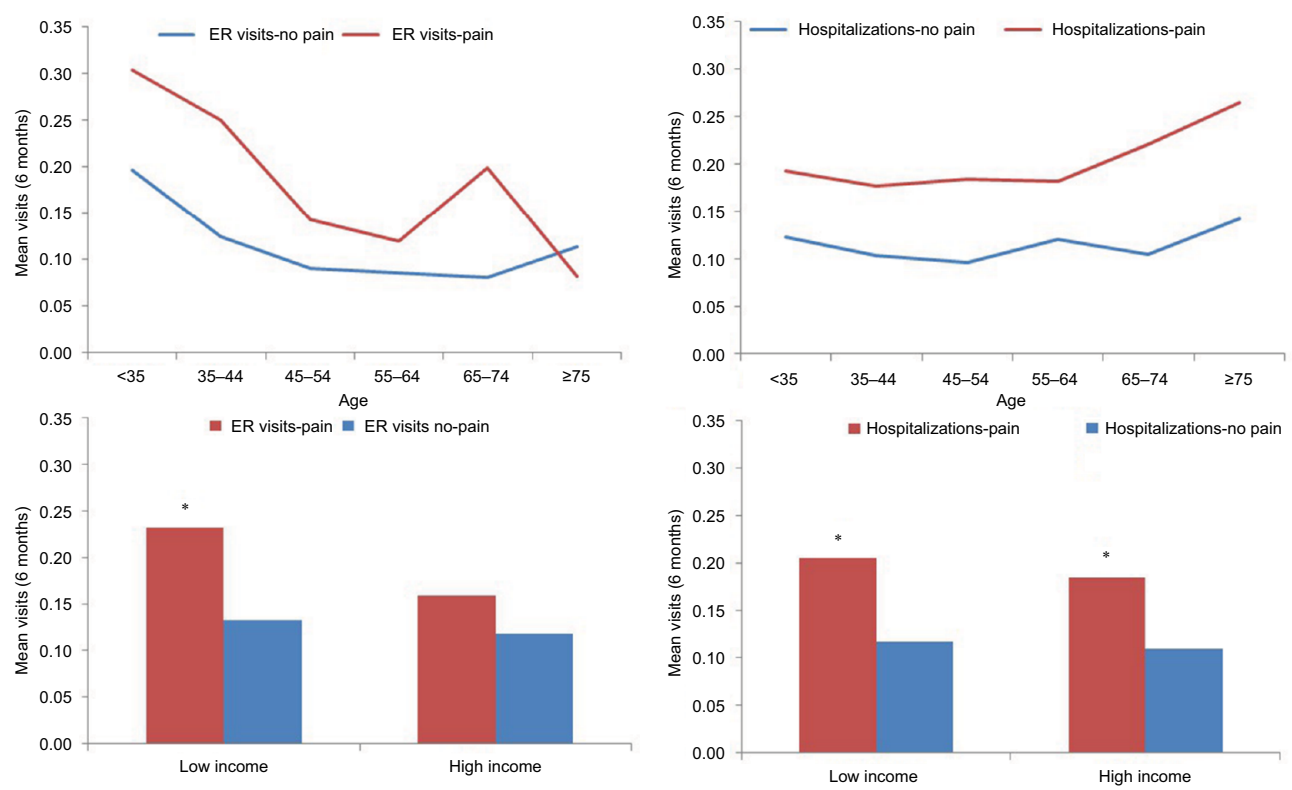

Figure I I Mean number of emergency room visits and hospitalizations in the past 6 months, according to pain, age group, and income level.

Notes: Pairwise tests between pain and no pain were significant for ER visits for ages $<35,35-44,45-54$, and $65-74$; for hospitalizations, for ages $<35$, 35-44, 45-54, 55-64, and $65-74 .{ }^{*} p<0.05$.

Abbreviation: ER, emergency room.
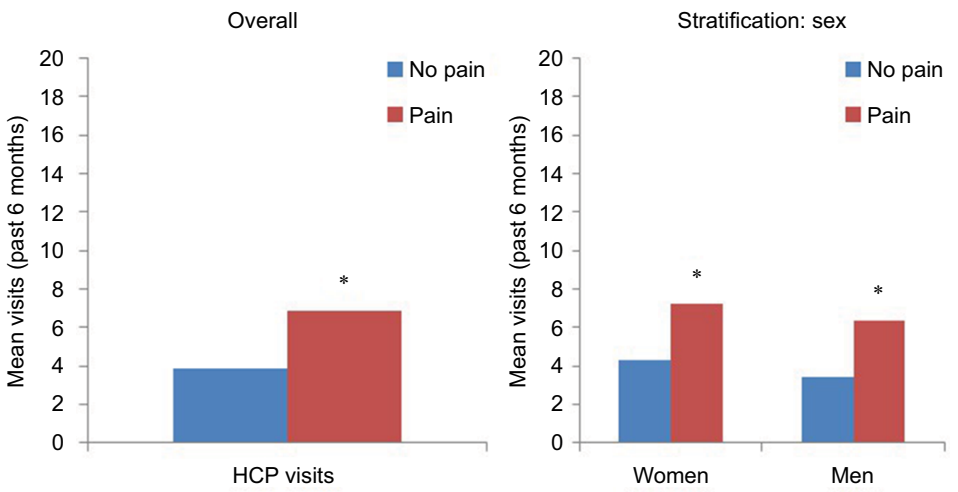

Stratification: $\mathrm{CCl}$

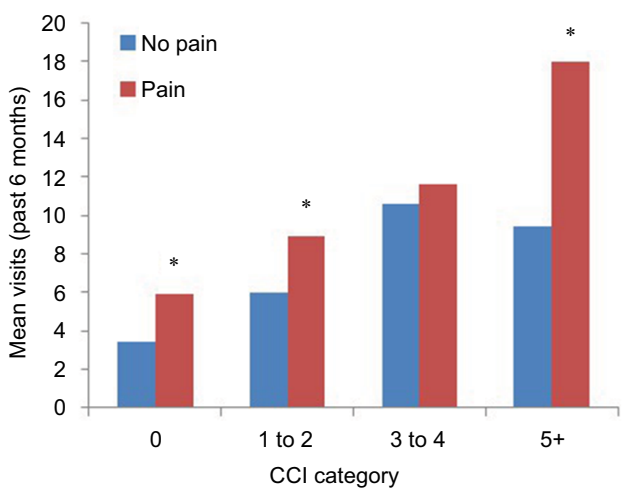

Figure 12 Health care provider visits (6 months, all-cause) by pain, sex, and $\mathrm{CCl}$ category.

Notes: Higher $\mathrm{CCl}$ categories had small sample sizes. ${ }^{*} p<0.05$.

Abbreviations: $\mathrm{CCl}$, Charlson comorbidity index 

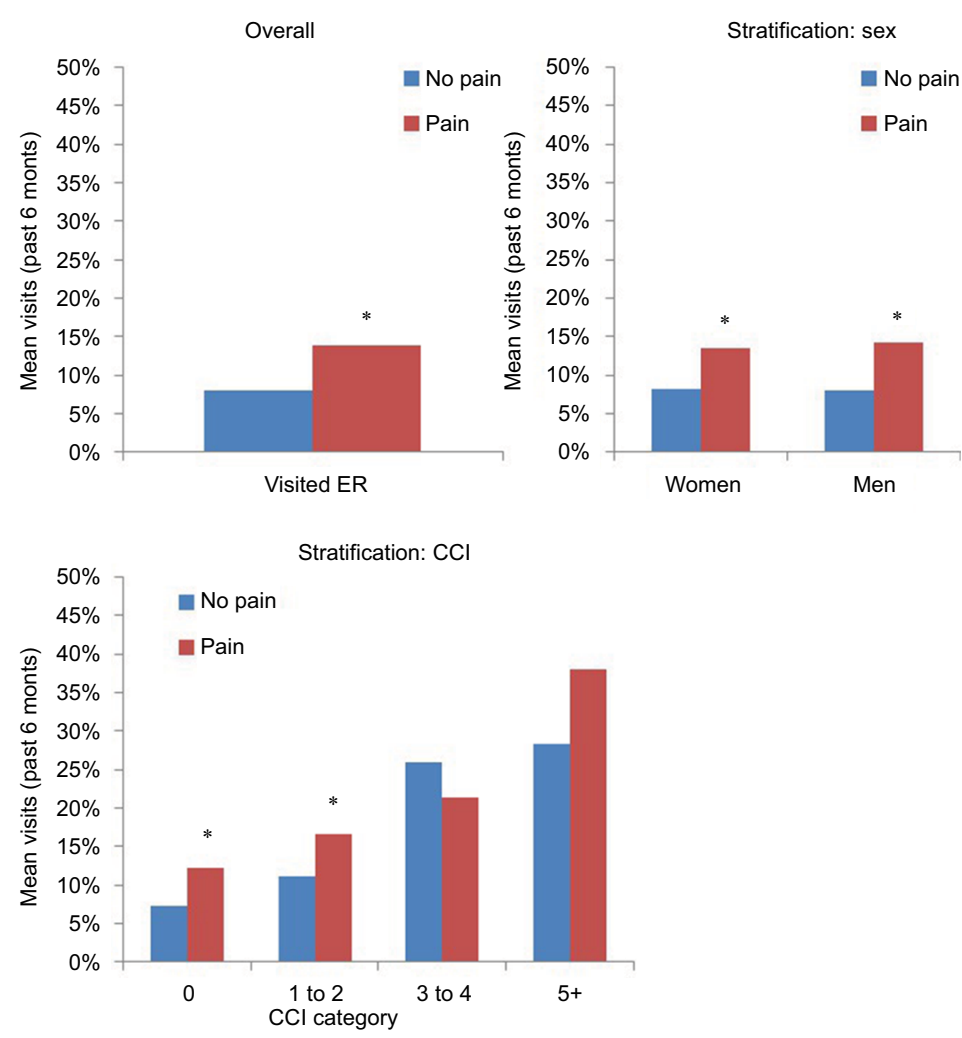

Figure 13 Percentage of adults visiting the emergency room by pain, sex, and $\mathrm{CCl}$ category.

Notes: Higher $\mathrm{CCl}$ categories had small sample sizes. ${ }^{*} p<0.05$.

Abbreviations: $\mathrm{CCl}$, Charlson comorbidity index.
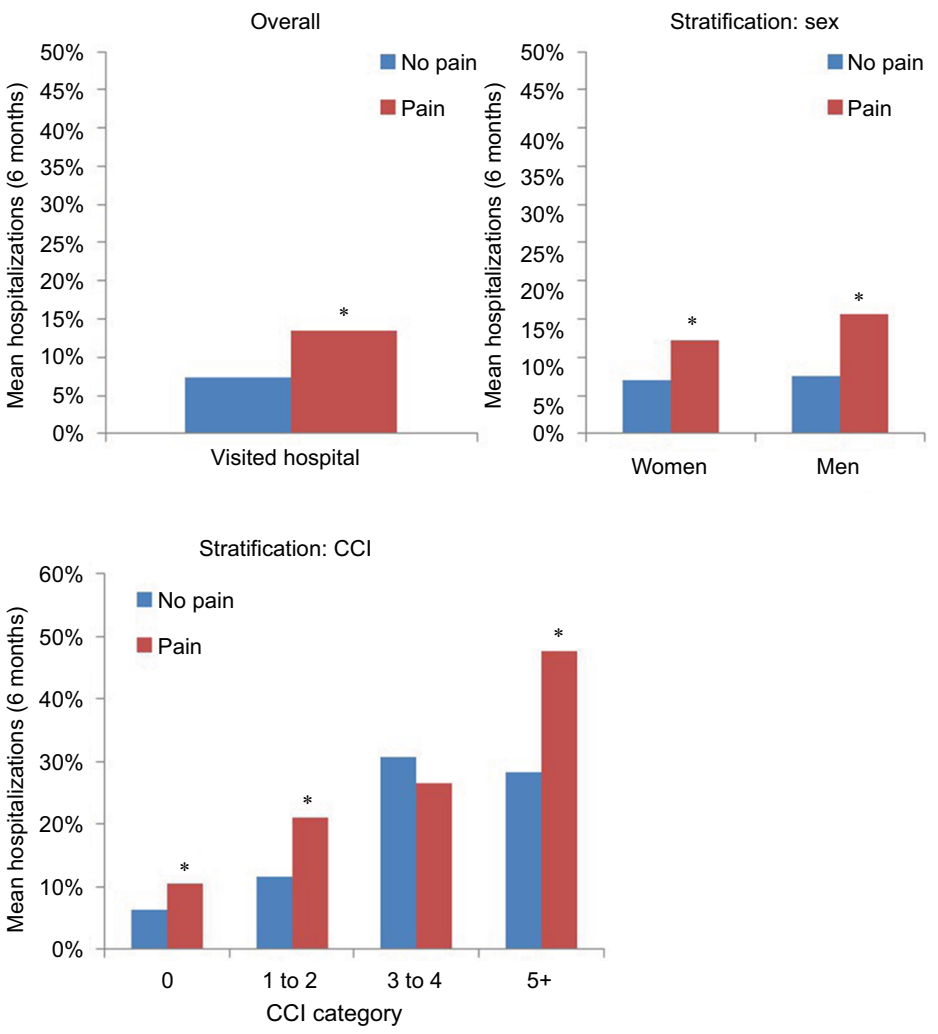

Figure 14 Percentage of adults who were hospitalized by pain, sex, and CCl category.

Notes: Higher $\mathrm{CCl}$ categories had small sample sizes. ${ }^{*} p<0.05$.

Abbreviation: $\mathrm{CCl}$, Charlson comorbidity index. 
The relationships between pain, age, and self-reported outcomes varied depending on the outcome in question. The mental health aspect of HRQoL was less affected by pain and increased across the lifespan, consistent with previous research..$^{13}$ The current study found that the prevalence of pain is highest in middle age, which is consistent with previous research and may potentially reflect reduced accessibility to treatment for older adults with pain conditions. ${ }^{917}$ Should this be the case, this suggests there is an unmet need for better pain management, especially among patients in this age group.

Even though previous research showed that female sex was related to a higher prevalence of pain, ${ }^{18}$ the results of the present study indicated that the relationships between pain and outcomes were generally consistent across sex. Taken together, this suggests that women may be more likely than men to experience pain, but among those who experience pain, there may be similar effects on HRQoL, employment, activity impairment, and HRU, irrespective of sex. The relationship between pain and impaired health outcomes was fairly stable across income groups, as pain (vs no pain) was associated with significantly lower HRQoL, regardless of income category. No differences in outcomes were found for those with (vs without) pain by level of comorbidity burden, although this finding should be evaluated with caution, given the small sample sizes of respondents in the higher-CCI categories.

\section{Limitations}

The present analysis had a number of limitations to consider. First, this study was cross-sectional, thereby precluding causal relationships from being established. All data were self-reported, and responses could not be independently validated. Furthermore, due to the self-reported nature of the data, the possibility that inaccuracies in recall or other response biases were introduced cannot be excluded.

While the NHWS was designed to reflect the age and sex distribution of the general adult population in France, it is unknown whether the study sample was representative of the subpopulation of adults who experience pain. For example, respondents who were in better health and/or in less pain may have been more likely to participate. If so, the current study may have underestimated the prevalence of pain and the magnitude of any differences between those who do and do not experience pain. Because the sample size of those with high CCI scores was very small, these analyses may have been underpowered to detect significant differences by comorbidity burden.
It is also possible that different results would have been obtained if relevant covariates (e.g., pain type, pain severity, psychiatric comorbidities, etc.) had been included in the analyses. However, given the focus of this study on examining absolute mean levels of pain and health outcomes among real-world respondents in France (as opposed to examining precise differences in outcomes), it was decided not to include multivariable models adjusting for covariates.

\section{Conclusion}

Pain is both common and burdensome among adults in France, with 1 in 5 experiencing pain in the past 12 months. The impact of pain was found to extend across subgroups, including age, income, sex, and level of comorbidity burden. Findings characterized both the scope of pain, as well as the nature of its accompanying burden, in France. Ultimately, this study, based on self-reported data, provides important information that can increase understanding of this considerable health and economic burden in order to encourage further investments in and development of pain management interventions in France.

\section{Acknowledgments}

The authors wish to acknowledge the assistance of Jeff Vietri, $\mathrm{PhD}$, with data analyses, Martine $\mathrm{C}$ Maculaitis, $\mathrm{PhD}$, with literature review and editing, and Amir Goren, $\mathrm{PhD}$, with editing. The assistance of JV, MCM, and AG was provided on behalf of Kantar Health, which received fees from Mundipharma SAS. The study reported on in this manuscript was funded by Mundipharma SAS.

\section{Disclosure}

$\mathrm{YH}, \mathrm{BC}$, and LG are employees of Mundipharma. The authors report no other conflicts of interest in this work.

\section{References}

1. Beck JG, Clapp JD. A different kind of co-morbidity: understanding posttraumatic stress disorder and chronic pain. Psychol Trauma. 2011;3(2):101-108.

2. Demyttenaere K, Bonnewyn A, Bruffaerts R, Brugh T, De Graaf $\mathrm{R}$, Alonso J. Comorbid painful physical symptoms and depression: prevalence, work loss, and help seeking. JAffect Disord. 2006;92(2-3): 185-193.

3. O'Brien T, Breivik H. The impact of chronic pain-European patients' perspective over 12 months. Scand J Pain. 2012;3:23-29.

4. Goren A, Mould-Quevedo J, DiBonaventura MD. Prevalence of pain reporting and associated health outcomes across emerging markets and developed countries. Pain Med. 2014;15(11):1880-1891.

5. Breivik H, Eisenberg E, O’Brien T. The individual and societal burden of chronic pain in Europe: the case for strategic prioritization and action to improve knowledge and availability of appropriate care. BMC Public Health. 2013;13:1229. 
6. Perrot S, Schaefer C, Knight T, Hufstader M, Chandran AB, Zlateva G. Societal and individual burden of illness among fibromyalgia patients in France: association between disease severity and OMERACT core domains. BMC Musculoskelet Disord. 2012;13:22.

7. Attal N, Lanteri-Minet M, Laurent B, Fermanian J, Bouhassira D. The specific disease burden of neuropathic pain: results of a French nationwide survey. Pain. 2011;152(12):2836-2843.

8. Depont F, Hunsche E, Abouelfath A, et al. Medical and non-medical direct costs of chronic low back pain in patients consulting primary care physicians in France. Fundam Clin Pharmacol. 2010;24(1):101-108.

9. Breivik H, Collett B, Ventafridda V, Cohen R, Gallacher D. Survey of chronic pain in Europe: prevalence, impact on daily life, and treatment. Eur J Pain. 2006;10(4):287-333.

10. Langley PC. The prevalence, correlates and treatment of pain in the European Union. Curr Med Res Opin. 2011;27(2):463-480.

11. Charlson ME, Pompei P, Ales KL, MacKenzie CR. A new method of classifying prognostic comorbidity in longitudinal studies: development and validation. J Chronic Dis. 1987;40(5):373-383.
12. Brazier JE, Roberts J. The estimation of a preference-based measure of health from the SF-12. Med Care. 2004;42(9):851-859.

13. Maruish ME, editor. New SF36v2 User Guide. 3rd ed. Lincoln: Quality Metric, Inc.; 2011.

14. Walters SJ, Brazier JE. Comparison of the minimally important difference for two health state utility measures: EQ-5D and SF-6D. Qual Life Res. 2005;14(6):1523-1532.

15. Ware JE, Kosinski M, Gandek B. SF-36 Health Survey: Manual and Interpretation Guide. 2nd ed. Lincoln: Quality Metric, Inc.; 2000.

16. Reilly MC, Zbrozek AS, Dukes EM. The validity and reproducibility of a work productivity and activity impairment instrument. Pharmacoeconomics. 1993;4(5):353-365.

17. Leadley RM, Armstrong N, Lee YC, Allen A, Kleijnen J. Chronic diseases in the European Union: the prevalence and health cost implications of chronic pain. J Pain Palliat Care Pharmacother. 2012;26(4):310-325.

18. Tsang A, Von Korff M, Lee S, et al. Common chronic pain conditions in developed and developing countries: gender and age differences and comorbidity with depression-anxiety disorders. J Pain. 2008;9(10):883-891.
ClinicoEconomics and Outcomes Research

\section{Publish your work in this journal}

ClinicoEconomics and Outcomes Research is an international, peerreviewed open-access journal focusing on health technology assessment, pharmacoeconomics and outcomes research in the areas of diagnosis, medical devices, and clinical, surgical and pharmacological intervention. The economic impact of health policy and health systems
Submit your manuscript here: https://www.dovepress.com/clinicoeconomics-and-outcomes-research-pourat

organization also constitute important areas of coverage. The manuscript management system is completely online and includes a very quick and fair peer-review system, which is all easy to use. Visit http://www.dovepress.com/testimonials.php to read real quotes from published authors. 